\title{
On the Uniqueness of the Infinite Cluster in the Percolation Model
}

\author{
A. Gandolfi ${ }^{1}$, G. Grimmett ${ }^{2}$ and L. Russo ${ }^{3}$ \\ 1 Department of Mathematics and Informatics, Delft University of Technology, Delft, The Netherlands \\ 2 School of Mathematics, University of Bristol, Bristol, England \\ ${ }^{3}$ Department of Mathematics, University of Rome II, Rome, Italy
}

\begin{abstract}
We simplify the recent proof by Aizenman, Kesten and Newman of the uniqueness of the infinite open cluster in the percolation model. Our new proof is more suitable for generalization in the direction of percolation-type processes with dependent site variables.
\end{abstract}

\section{Introduction}

It has long been conjectured that the infinite open cluster of the percolation model is unique (almost surely) whenever it exists. This conjecture was verified affirmatively in the recent paper of Aizenman, Kesten and Newman [1], which is couched in the context of (possibly long-range) percolation on any lattice $\mathscr{L}$. Their proof utilizes several distinct ideas and techniques, some of which have their origins in statistical mechanics. Furthermore, the proof has certain consequences for the "thermodynamic functions" of percolation theory, such as the number of clusters per site and the connectivity functions. On the other hand, there are certain miraculous aspects to the method of proof in [1], and it was in attempting to understand this proof that the ideas of this note evolved. In this note, we present a proof of the uniqueness of the infinite open cluster which uses essentially only one of the main ingredients of [1], namely a large-deviation estimate for a certain random variable defined on large but finite open clusters.

The principal motivation for this work was to understand how one may prove the uniqueness theorem for more general processes than "Bernoulli" percolation. Such a generalization to a class of Gibbs measures will appear in [3]. In addition, we hope that our argument may be useful in approaching the question of the uniqueness of the "incipient infinite cluster" of the percolation process; see [2] and [8]. In related work, Gandolfi, Keane and Russo [4] have shown that the infinite cluster is unique for a certain class of two-dimensional models; their techniques are similar to those of Harris [6] for Bernoulli percolation, and are quite different from the general arguments of [1] and the present paper.

We refer the reader to [1] and [4] for motivation and background. 


\section{The Result}

We state and prove the result for site percolation on $\mathbb{Z}^{d}$, where $d \geqq 2$, but the same proof is valid for all bond, site, and mixed bond/site models as well as long-range bond models.

Let $\mu_{p}$ be the Bernoulli product measure on the configuration space $\{-1,1\}^{\mathbb{Z}^{d}}$ with density $p$, where $0<p<1$. We denote by $E_{p}$ the expectation operator related to $\mu_{p}$, and we call a site open if its state is 1 and closed otherwise. Writing $I$ for the event that there exists an infinite open cluster, we shall prove the following result.

Theorem. If $p$ is such that $\mu_{p}(I)>0$, then there exists almost surely a unique infinite open cluster.

Proof. We fix a large positive integer $n$, and let $\Lambda$ be the box $\left\{x \in \mathbb{Z}^{d}:\left|x_{i}\right| \leqq n\right.$ for all $i\}$. Later we shall take the limit as $n \rightarrow \infty$; we express this limit as the limit "as $\Lambda \rightarrow \infty$ ". For $x \in \Lambda$, we write $C(x)$ for the open cluster of $\Lambda$ containing $x$, and $\mathscr{C}_{\Lambda}$ for the set of all open clusters of $\Lambda$ which intersect the internal boundary $\left\{x \in \mathbb{Z}^{d}:\left|x_{i}\right|=n\right.$ for some $\left.i\right\}$ of $\Lambda$. We consider the following random subsets of $\Lambda$ :

$$
\begin{aligned}
& F_{\Lambda}(\omega)=\bigcup_{C \in \mathscr{C}_{\Lambda}} C ; \quad G_{\Lambda}(\omega)=\bigcup_{C \in \mathscr{C}_{\Lambda}} \partial C ; \\
& H_{\Lambda}(\omega)=\bigcup_{\substack{C_{1}, C_{2} \in \mathscr{C}_{\Lambda} \\
C_{1} \neq C_{2}}}\left\{\partial C_{1} \cap \partial C_{2}\right\} .
\end{aligned}
$$

Here, $\partial C$ represents the external boundary (in $\Lambda$ ) of $C$, being the set of sites of $\Lambda$ which do not belong to $C$ but which are adjacent to some site in $C$. Thus $F_{\Lambda}$ is the set of sites which are joined by open paths to the internal boundary of $\Lambda, G_{\Lambda}$ is the set of closed sites which are adjacent to sites in $F_{\Lambda}$, and $H_{\Lambda}$ is the set of closed sites which are adjacent to sites in two or more of the clusters in $\mathscr{C}_{\Lambda}$. We write $L_{x}$ for the event that the site $x$ belongs to the external boundary of two or more infinite open clusters of the lattice. It is easy to see that

$$
\lim _{\Lambda \rightarrow \infty} \frac{1}{|\Lambda|} E_{p}\left(\left|H_{\Lambda}\right|\right)=\mu_{p}\left(L_{x}\right)
$$

where $|A|$ is the cardinality of the set $A$. On the other hand (see [1] and [9]), the number of infinite open clusters equals 0 or 1 almost surely if $\mu_{p}\left(L_{x}\right)=0$, and so it suffices to prove that

$$
\limsup _{\Lambda \rightarrow \infty} \frac{1}{|\Lambda|} E_{p}\left(\left|H_{\Lambda}\right|\right) \leqq 0
$$

the remainder of the proof is devoted to showing this.

From the definition of $G_{\Lambda}$ and $H_{\Lambda}$, we have that

$$
\left|H_{\Lambda}(\omega)\right| \leqq\left(\sum_{C \in \mathscr{C}_{\Lambda}}|\partial C|\right)-\left|G_{\Lambda}(\omega)\right| .
$$

On the other hand,

$$
\mu_{p}\left(x \in F_{\Lambda} \mid x \in F_{\Lambda} \cup G_{\Lambda}\right)=p
$$


if $x$ is not a boundary vertex of $\Lambda$, so that

$$
E_{p}\left(\sum_{C \in \mathscr{C}_{\Lambda}}|C|\right)=E_{p}\left|F_{\Lambda}\right|=\frac{p}{1-p} E_{p}\left|G_{\Lambda}\right|+o(|\Lambda|),
$$

giving from (3) that

$$
\frac{1}{|\Lambda|} E_{p}\left(\left|H_{\Lambda}\right|\right) \leqq \frac{1}{|\Lambda|} E_{p}\left(\sum_{C \in \mathscr{E}_{\Lambda}}\left\{|\partial C|-\frac{1-p}{p}|C|\right\}\right)+o(1) .
$$

In order to estimate the right-hand side here, we require (as in [1]) the large-deviation estimate

$$
\mu_{p}(h(C(x)) \geqq \varepsilon k,|\bar{C}(x)|=k) \leqq \exp \left(-\frac{k \varepsilon^{2}}{4 a}\right),
$$

valid for all $x \in \Lambda, k \geqq 1$, and $\varepsilon>0$, where $h(C(x))=|\partial C(x)|-p^{-1}(1-p)|C(x)|$, $\bar{C}(x)=C(x) \cup \partial C(x)$, and $a=a(p)$ is a function of $p$ which is strictly positive on $(0,1)$. For the convenience of the reader we include a proof of $(6)$, although this proof differs only slightly from the corresponding step in [1]; these differences arise from the facts that we are considering clusters of $\Lambda$ only, and we need only an estimate which is one-sided in $h(C(x))$. Let $a_{m \ell}(x)$ be the number of connected subgraphs of $\Lambda$ with $m$ sites in their interiors and $\ell$ sites in their external boundaries and which contain $x$. For any $r \geqq 0$, we have that

$$
\begin{aligned}
\mu_{p}(h(C(x)) \geqq \varepsilon k,|\bar{C}(x)|=k) & \leqq e^{-\varepsilon k r} E_{p}\left(e^{r h(C(x))} ;|\bar{C}(x)|=k\right) \\
& =e^{-\varepsilon k r} \sum_{\substack{m, \ell \\
m+\ell=k}} a_{m \ell}(x)\left(p e^{-r p^{-1}(1-p)}\right)^{m}\left\{(1-p) e^{r}\right\}^{\ell} \\
& \leqq e^{-\varepsilon k r} f(r, p)^{k} \mu_{\pi}(|\bar{C}(x)|=k),
\end{aligned}
$$

where

$$
f(r, p)=p e^{-r p^{-1}(1-p)}+(1-p) e^{r},
$$

and $\pi=p e^{-r p^{-1}(1-p)} / f(r, p)$. Now $f(r, p)=1+O\left(r^{2}\right)$ as $r \downarrow 0$, so that

$$
\mu_{p}(h(C(x)) \geqq \varepsilon k,|\bar{C}(x)|=k) \leqq e^{-\varepsilon k r+a k r^{2}}
$$

for some function $a=a(p)$ which is strictly positive and finite on $(0,1)$. We choose $r$ to minimize the right-hand side of (7) and obtain (6).

Finally we show that (5) and (6) imply (2). We fix $\varepsilon>0$ and $\delta$ such that $0<\delta<1 / d$, and we write

$$
\mathscr{C}_{\Lambda}^{\prime}(\omega)=\left\{C \in \mathscr{C}_{\Lambda}(\omega):|\bar{C}| \geqq|\Lambda|^{\delta}\right\}
$$

and $B_{\Lambda}$ for the event that $h(C)=|\partial C|-p^{-1}(1-p)|C| \leqq \varepsilon|\bar{C}|$ for all $C \in \mathscr{C}_{\Lambda}^{\prime}$. From (6),

$$
1-\mu_{p}\left(B_{\Lambda}\right) \leqq|\Lambda| \sum_{k=|\Lambda|^{\delta}}^{\infty} \exp \left(-\frac{k \varepsilon^{2}}{4 a}\right) \rightarrow 0 \quad \text { as } \quad \Lambda \rightarrow \infty,
$$

since, if $B_{\Lambda}$ does not occur, then there exists a site $x$ in $\Lambda$ such that $h(C(x))>\varepsilon|\bar{C}(x)|$ 
and $|\bar{C}(x)| \geqq|\Lambda|^{\delta}$. Thus

$$
\frac{1}{|\Lambda|} E_{p}\left(\sum_{C \in \mathscr{G}_{\Lambda}^{\prime}}\left\{|\partial C|-\frac{1-p}{p}|C|\right\}\right) \leqq\left(\frac{1}{|\Lambda|} E_{p} \sum_{C \in \mathscr{G}_{\Lambda}^{\prime}} \varepsilon|\bar{C}|\right)+2 d\left\{1-\mu_{p}\left(B_{\Lambda}\right)\right\} \rightarrow 0,
$$

as $\Lambda \rightarrow \infty$ and $\varepsilon \downarrow 0$. On the other hand, any cluster in $\mathscr{C}_{\Lambda}(\omega) \backslash \mathscr{C}_{\Lambda}^{\prime}(\omega)$ is contained in a $d$-dimensional "annulus" of $\Lambda$ with thickness of order $|\Lambda|^{\delta}$, giving that

$$
\lim _{\Lambda \rightarrow \infty} \frac{1}{|\Lambda|} E_{p}\left(\sum_{C \in \mathscr{E}_{\Lambda} \backslash \mathscr{C}_{\Lambda}^{\prime}}\left\{|\partial C|-\frac{1-p}{p}|C|\right\}\right)=0 .
$$

We combine this with (5) and (9) to deduce (2), and the proof is complete.

We note that similar calculations appear in [5], and a related approach to the large-deviation argument may be found in [7].

Acknowledgements. Some of the ideas contained in this note were developed during visits of L.R. to Delft University of Technology and G.G. to Cornell University, the latter being with the aid of a Fulbright Travel Grant. We are indebted to Harry Kesten for many valuable discussions on this topic and related subjects.

\section{References}

1. Aizenman, M., Kesten, H., Newman, C. M.: Uniqueness of the infinite cluster and continuity of connectivity functions for short and long range percolation. Commun. Math. Phys. 111, 505-531 (1987)

2. Coniglio, A.: Shapes, surfaces and interfaces in percolation clusters. In: Physics of finely divided matter. Daoud, M. (ed.). Les Houches Session XLIV 1985 (to appear)

3. Gandolfi, A.: in preparation

4. Gandolfi, A., Keane, M., Russo, L.: On the uniqueness of the infinite occupied cluster in dependent two-dimensional site percolation. Preprint 1986

5. Grimmett, G. R.: On the differentiability of the number of clusters per vertex in the percolation model. J. Lond. Math. Soc. 23, 372-384 (1981)

6. Harris, T. E.: A lower bound for the critical probability in a certain percolation process. Proc. Camb. Phil. Soc. 56, 13-20 (1960)

7. Kesten, H.: Percolation theory for mathematicians. Boston: Birkhäuser 1982

8. Kesten, H.: The incipient infinite cluster in two-dimensional percolation. Probab. Theory Related Fields 73, 369-394 (1986)

9. Newman, C. M., Schulman, L. S.: Infinite clusters in percolation models. J. Stat. Phys. 26, 613-628 (1981)

Communicated by M. E. Fisher

Received May 12, 1987; in revised form August 27, 1987 